\title{
化学与生物转化法合成帕立骨化醇
}

\author{
万阳 文 鹏张攀陆 群* \\ (西南交通大学生命科学与工程学院 成都 610031)
}

\begin{abstract}
摘要 以维生素 $\mathrm{D}_{2}$ (1) 为原料, 通过七步有机合成反应和一次生物转化, 成功获得了帕立骨化醇 $\mathbf{9}$, 并得到了一条新的 合成帕立骨化醇的路线, 收率为 $3.24 \%$. 该合成方法路线短, 操作简便, 收率较文献中的方法有较大提高. 所有产物结 构经 ${ }^{1} \mathrm{H}$ NMR, ${ }^{13} \mathrm{C}$ NMR 和 MS 表征.
\end{abstract}

关键词 维生素 $\mathrm{D}_{2}$; 美基化; 帕立骨化醇

\section{Novel Synthesis of Paricalcitol by Chem-biotransformation}

\author{
Wan, Yang Wen, Peng Zhang, Pan Lu, Qun* \\ (School of Life Science and Engineering, Southwest Jiaotong University, Chengdu 610031)
}

\begin{abstract}
Paricalcitol (9) was obtained from Vitamin $\mathrm{D}_{2}$ via a route of the combination of seven-step organic reaction and biotransformation. The overall yield was $3.24 \%$, which was higher than those of the reported methods. The structures of all intermediates and product were confirmed by ${ }^{1} \mathrm{H}$ NMR, ${ }^{13} \mathrm{C}$ NMR and MS techniques.

Keywords vitamin $\mathrm{D}_{2}$; hydroxylation; paricalcitol
\end{abstract}

维生素 D 的生物活性形式骨化三醇(calcitol)是调节 体内钻磷代谢平衡的激素. 近年来, 其在激素(甲状旁 腺激素, PTH)分泌、细胞增殖分化以及免疫调节等一系 列生理功能中所起的关键作用逐渐被人们认识 ${ }^{[1,2]}$, 也 预示着其在相关领域的治疗潜能. 然而受其高钲磷调节 活性的影响, 骨化三醇在作为 PTH 分泌抑制剂、抗细胞 增殖剂或免疫调节剂使用时常常会发生高血钙的毒副 作用. 鉴于骨化三醇在临床使用时的上述局限性, 已对 骨化三醇做了大量化学结构修饰 ${ }^{[3]}$, 以使其钙调活性与 其他活性相分离. 由雅培公司(Abbott)开发的活性维生 素 D 类似物帕立骨化醇 ${ }^{[4,5]}$ 能够选择性降低慢性肾病 (CKD) 患者血浆甲状旁腺激素 $(\mathrm{PTH})$ 水平, 而极少引起 高钙血症 ${ }^{[6]}$, 以其为活性成分的商品 Zemplar 注射剂及 胶囊分别于 1998 年和 2005 年上市, 是目前应用范围最 广的 CKD 患者继发性甲状旁腺功能六进症(SHPT)的预 防及治疗药物.

关于帕立骨化醇的合成已经有许多报道 ${ }^{[7 ~ 13]}$. 文献 $[7,8]$ 采用的是全合成方法, 由来源于维生素 $\mathrm{D}_{2}$ 、奎宁酸
和 2-甲基-3-羊基丙酸甲酯的三个合成子汇聚合成得到 帕立骨化醇, 由于全合成方法需要分别合成 $\mathrm{A}$ 环和 $\mathrm{C} / \mathrm{D}$ 环合成子, 合成路线长, 产率低, 不利于大量生产. 以 容易获得的维生素 $\mathrm{D}_{2}$ 为前体进行结构修饰的方法缩短 了合成路线, 因此更具有实际生产意义. 文献[9１1]采 用的是全化学合成法, 需要通过侧链断裂后再形成来实 现 25 位羟基化, 但需要多步基团保护和脱保护, 使得步 骤繁琐, 收率较低. 文献[12, 13]以 25-羟基-维生素 $\mathrm{D}_{2}$ 为原料, 前者通过烯丙位氧化反应实现 $1 \alpha$ 羊基化, 然后 通过双差基化、氧化断键和羰基还原实现 19 位去甲基, 而后者也通过双羟基化和氧化断键，然后对中间体烯烃 的硼氢化反应实现 $1 \alpha$ 位羟基化. 但二者的主要缺点是 初始原料 25-羟基-维生素 $\mathrm{D}_{2}$ 缺乏商业来源, 难以大量 获得 ${ }^{[11]}$. 本研究从更易获得的维生素 $\mathrm{D}_{2}$ 出发, 而不是 文献[12，13]所采用的 25-羊基-维生素 $\mathrm{D}_{2}$, 通过化学合 成获得 $1 \alpha$-差基-19-去甲维生素 $\mathrm{D}_{2}$ 后利用生物转化法实 现 25 位羟基化, 所采用菌株为本实验室篮选所得 CGMCC 5098 放线菌. 实验路线见 Scheme 1.

\footnotetext{
*E-mail: luqun1125@126.com

Received April 12, 2012; revised June 6, 2012; published online June 19, 2012.

Project supported by the Science and Technology Support Fundation of Sichuan Province (No. 2010SZ124).

四川省科技支撑基金(No. 2010SZ124)资助项目.
} 

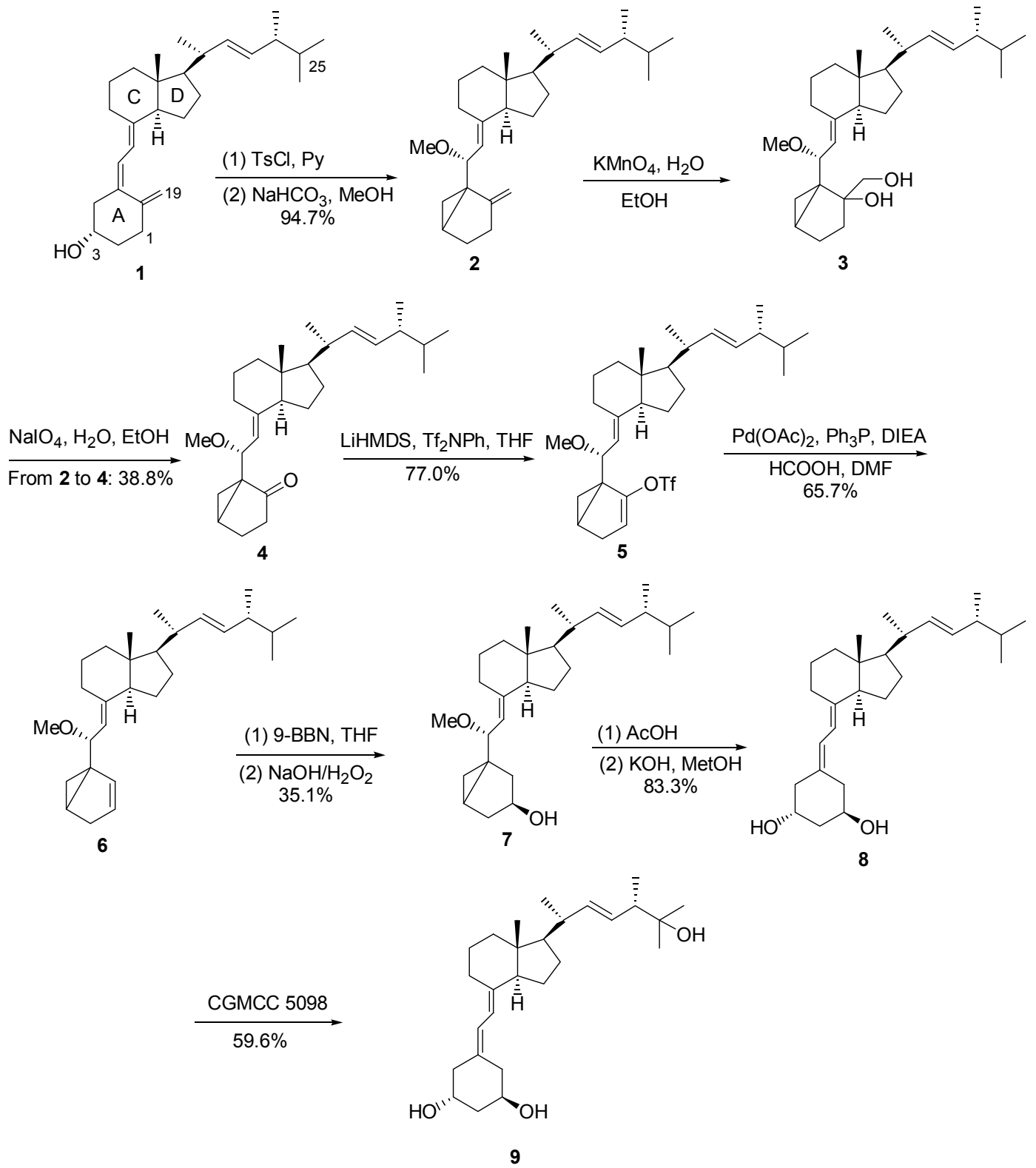

Scheme 1

\section{1 实验部分}

\section{1 仪器及试剂}

${ }^{1} \mathrm{H}$ NMR 与 ${ }^{13} \mathrm{C}$ NMR 用 Varian INOVA- 400 型核磁 共振仪测定, TMS 为内标; MS 使用 Waters Quattro Premier XE Mass Spectrometer 型质谱仪测定, 甲醇为溶剂. 所用试剂均为国产(或进口)化学纯或分析纯.

\subsection{6-甲氧基-3,5-环维生素 $\mathrm{D}_{2}$ (2)的合成}

将维生素 $\mathrm{D}_{2}$ (1) $10.00 \mathrm{~g}(25.25 \mathrm{mmol})$ 和对甲苯磺酰 氯 $12 \mathrm{~g}(63.16 \mathrm{mmol})$ 加入反应瓶中, 用 $50 \mathrm{~mL}$ 吡啶完全 溶解, 在恒温冷冻浴槽中 $\left(2 \sim 4{ }^{\circ} \mathrm{C}\right)$ 搅拌 $48 \mathrm{~h}$. TLC [ $V$ (石 油醚) $: V$ (乙酸乙酯 $)=5: 1$ 监测至反应完全. 加入 200 $\mathrm{mL}$ 饱和碳酸氢钠溶液, 立即有大量气泡产生, 并有固
体析出，过滤，固体用 $200 \mathrm{~mL} 3 \%$ 盐酸洗涤，收集固体 成分, 晾干. 将所得干燥固体和碳酸氢钠固体 $(50 \mathrm{~g})$ 加 入反应瓶, 用 $500 \mathrm{~mL}$ 甲醇完全溶解, $60{ }^{\circ} \mathrm{C}$ 搅拌回流 5 6 h. TLC $[V$ (石油醚 $) ： V($ 乙酸乙酯 $)=10 ： 1]$ 监测至反应 完全. 减压回收甲醇, 向瓶中加入 $200 \mathrm{~mL}$ 水, 反应液依 次用乙酸乙酯萃取 $(200 \mathrm{~mL} \times 3)$, 饱和食盐水洗 $(200$ $\mathrm{mL}$ ), 无水硫酸钠干燥, 浓缩, 得到棕色油状物 $9.80 \mathrm{~g} 2$, 收率为 $94.7 \%$. 此粗品可直接用于下步反应. ${ }^{1} \mathrm{H}$ NMR $\left(\mathrm{CDCl}_{3}, 400 \mathrm{MHz}\right) \delta: 5.23 \sim 5.13(\mathrm{~m}, 2 \mathrm{H}, \mathrm{H}-22, \mathrm{H}-23)$, 5.03 (brs, 1H, H-19a), 4.98 (d, $J=9.2 \mathrm{~Hz}, 1 \mathrm{H}, \mathrm{H}-7$ ), 4.87 (brs, 1H, H-19b), 4.16 (d, J=9.2 Hz, 1H, H-6), 3.24 (s, $3 \mathrm{H}, \mathrm{OMe}$ ), $2.68 \sim 2.60(\mathrm{~m}, 1 \mathrm{H}, \mathrm{H}-9 \mathrm{a}), 2.28 \sim 2.20(\mathrm{~m}, 1 \mathrm{H})$, $2.12 \sim 1.95(\mathrm{~m}, 4 \mathrm{H}), 1.87 \sim 1.25(\mathrm{~m}, 14 \mathrm{H}), 1.01(\mathrm{~d}, J=6.8$ 
$\mathrm{Hz}, 3 \mathrm{H}, \mathrm{H}-21), 0.91$ (d, $J=6.8 \mathrm{~Hz}, 3 \mathrm{H}, \mathrm{H}-28), 0.87 \sim 0.80$ (m, 7H, H-4a, H-26, H-27), 0.73 (t, $J=4 \mathrm{~Hz}, 1 \mathrm{H}, \mathrm{H}-4 \mathrm{~b}$ ), 0.54 (s, $3 \mathrm{H}, \mathrm{H}-18) ;{ }^{13} \mathrm{C}$ NMR $\left(\mathrm{CDCl}_{3}, 100 \mathrm{MHz}\right) \delta: 152.1$, 143.3, 135.4, 131.8, 119.0, 103.8, 77.3, 56.3, 55.9, 45.2, $42.7,40.3,40.2,35.4,33.0,30.1,29.7,29.3,27.7,25.3$, 24.2, 23.5, 22.0, 21.0, 19.9, 19.5, 17.5, 16.3, 12.0; ESI-MS $m / z: 433.3[\mathrm{M}+\mathrm{Na}]^{+}$.

\subsection{0,19-双羟基-6-甲氧基-3,5-环维生素 $\mathrm{D}_{2}$ (3)的合 成}

将 $2(5.00 \mathrm{~g}, 12.20 \mathrm{mmol})$ 加入反应瓶，用 $500 \mathrm{~mL}$ 乙 醇完全溶解, 加入 $200 \mathrm{~mL} 3 \% \mathrm{KMnO}_{4}$ 水溶液 (3.11 equiv.). $-20{ }^{\circ} \mathrm{C}$ 搅拌反应 $2 \sim 3 \mathrm{~h}$. TLC [ $V$ (石油醚) : $V($ 乙酸乙酯 $)=4: 1$ ] 监测至反应完全. 将反应液于 $40{ }^{\circ} \mathrm{C}$ 水浴搅拌 $0.5 \mathrm{~h}$, 过滤, 减压回收乙醇, 反应液依次用乙 酸乙酯萃取 $(200 \mathrm{~mL} \times 3)$, 饱和食盐水洗涤 $(200 \mathrm{~mL})$, 无 水硫酸钠干燥, 浓缩, 得到棕色油状物 $5.75 \mathrm{~g} \mathrm{3}$. 此粗品 可直接用于下步反应. ${ }^{1} \mathrm{H}$ NMR $\left(\mathrm{CDCl}_{3}, 400 \mathrm{MHz}\right) \delta$ : $5.19 \sim 5.08(\mathrm{~m}, 2 \mathrm{H}, \mathrm{H}-22, \mathrm{H}-23), 4.78(\mathrm{~d}, J=9.6 \mathrm{~Hz}, 1 \mathrm{H}$, H-6), 4.51 (d, $J=9.6 \mathrm{~Hz}, 1 \mathrm{H}, \mathrm{H}-7), 3.64$ (d, $J=10.8 \mathrm{~Hz}$, 1H, H-19a), 3.53 (d, $J=10.8 \mathrm{~Hz}, 1 \mathrm{H}, \mathrm{H}-19 \mathrm{~b}$ ), 3.18 (s, 3H, OMe), $2.56 \sim 2.46(\mathrm{~m}, 1 \mathrm{H}, \mathrm{H}-9 \mathrm{a}), 2.03 \sim 1.89(\mathrm{~m}, 4 \mathrm{H})$, $1.84 \sim 1.77(\mathrm{~m}, 1 \mathrm{H}), 1.65 \sim 1.58(\mathrm{~m}, 4 \mathrm{H}), 1.52 \sim 1.37(\mathrm{~m}$, $6 \mathrm{H}), 1.33 \sim 1.21(\mathrm{~m}, 4 \mathrm{H}), 1.19 \sim 1.09(\mathrm{~m}, 1 \mathrm{H}), 0.97(\mathrm{~d}, J=$ $6.4 \mathrm{~Hz}, 3 \mathrm{H}, \mathrm{H}-21), 0.87$ (d, $J=6.4 \mathrm{~Hz}, 3 \mathrm{H}, \mathrm{H}-28), 0.79 \sim$ 0.76 (m, 6H, H-26, H-27), 0.49 (s, 3H, H-18), 0.42 (t, $J=$ $6.4 \mathrm{~Hz}, 1 \mathrm{H}, \mathrm{H}-4 \mathrm{~b}) ;{ }^{13} \mathrm{C} \mathrm{NMR}\left(\mathrm{CDCl}_{3}, 100 \mathrm{MHz}\right) \delta: 145.6$, 135.3, 131.9, 117.0, 83.5, 78.8, 67.6, 56.2, 55.7, 55.4, 45.1, $42.7,40.2,39.9,35.2,33.1,32.9,28.9,27.6,24.3,23.3$, 22.1, 21.0, 20.3, 19.9, 19.5, 17.5, 12.1, 11.7; ESI-MS m/z: $467.3[\mathrm{M}+\mathrm{Na}]^{+}$.

\subsection{0-酮-6-甲氧基-3,5-环维生素 $\mathrm{D}_{2}$ (4)的合成}

将粗品 $3(5.75 \mathrm{~g}, 12.95 \mathrm{mmol})$ 加入反应瓶, 用 100 $\mathrm{mL}$ 乙醇完全溶解, 加入 $50 \mathrm{~mL} 10 \%$ 高碘酸钠水溶液 (1.81 equiv.), 冰浴摚拌 $1 \mathrm{~h}$. TLC [ $V$ (石油醚)： $V$ (乙酸乙 酯 $)=4: 1$ ] 监测至反应完全. 将反应液过滤, 减压回收 乙醇, 滤液依次用乙酸乙酯萃取 $(100 \mathrm{~mL} \times 3)$, 饱和食盐 水洗涤 $(200 \mathrm{~mL})$, 无水硫酸钠干燥, 浓缩, 硅胶柱层析 分离 $[V$ (石油醚) $: V($ 乙酸乙酯 $)=12: 1]$ 得到淡黄色油状 物 $1.95 \mathrm{~g} \mathrm{4}$, 二步收率为 $38.8 \% .{ }^{1} \mathrm{H}$ NMR $\left(\mathrm{CDCl}_{3}, 400\right.$ MHz) $\delta: 5.24 \sim 5.13$ (m, 2H, H-22, H-23), 4.73 (d, $J=9.6$ Hz, 1H, H-6), 4.56 (d, J=9.6 Hz, 1H, H-7), 3.23 (s, 3H, $\mathrm{OMe}), 2.70 \sim 2.58(\mathrm{~m}, 1 \mathrm{H}, \mathrm{H}-9 \mathrm{a}), 2.18 \sim 2.10(\mathrm{~m}, 3 \mathrm{H})$, $2.04 \sim 1.94(\mathrm{~m}, 4 \mathrm{H}), 1.88 \sim 1.83(\mathrm{~m}, 1 \mathrm{H}), 1.74 \sim 1.67(\mathrm{~m}$, $4 \mathrm{H}), 1.51 \sim 1.21(\mathrm{~m}, 7 \mathrm{H}), 1.02(\mathrm{~d}, J=6.4 \mathrm{~Hz}, 3 \mathrm{H}, \mathrm{H}-21)$, 0.92 (d, $J=6.8 \mathrm{~Hz}, 3 \mathrm{H}, \mathrm{H}-28), 0.89 \sim 0.81$ (m, 8H, H-26,
$\mathrm{H}-27, \mathrm{H}-4 \mathrm{a}, \mathrm{H}-4 \mathrm{~b}), 0.53$ (s, 3H, H-18); ${ }^{13} \mathrm{C}$ NMR $\left(\mathrm{CDCl}_{3}\right.$, $100 \mathrm{MHz}) \delta: 214.1,145.3,135.4,131.9,116.9,72.8,56.2$, $53.8,45.1,42.7,40.7,40.3,40.0,33.0,32.9,29.2,27.6$, 23.9, 23.3, 22.0, 21.4, 21.0, 19.9, 19.6, 17.5, 17.0, 12.1; ESI-MS $m / z: 435.2[\mathrm{M}+\mathrm{Na}]^{+}$.

\subsection{6-甲氧基-10-三氟甲磺酰-3,5-环维生素 $\mathrm{D}_{2}$ (5)的合 成}

将 $4(1.95 \mathrm{~g}, 4.73 \mathrm{mmol})$ 加入反应瓶中, 氮气保护 下, 注入 $1.8 \mathrm{~mL}$ 无水四氢呋喃使其完全溶解, 然后注入 LiHMDS ( $1 \mathrm{~mol} / \mathrm{L}$ in THF) $9 \mathrm{~mL}$ (1.90 equiv.), $-78{ }^{\circ} \mathrm{C}$ 搅拌 $1 \mathrm{~h}$. 加入用 $5 \mathrm{~mL}$ 无水四氢呋喃溶解的 $\mathrm{Tf}_{2} \mathrm{NPh} 3.04$ $\mathrm{g}$ (1.80 equiv.), 继续低温 $\left(-78 \sim-15{ }^{\circ} \mathrm{C}\right.$, 缓慢升温)摚 拌 4 5 h. TLC [ $V$ (石油醚)：V(乙酸乙酯 $)=10 ： 1$ ] 监测 至反应完全. 减压回收四氢呋喃, 向瓶中加入 $50 \mathrm{~mL}$ 水, 依次用乙酸乙酯萃取 $(50 \mathrm{~mL} \times 3), 3 \%$ 盐酸 $(50 \mathrm{~mL} \times 2)$ 、 饱和碳酸氢钠 $(50 \mathrm{~mL} \times 2)$ 、饱和食盐水洗涤 $(50 \mathrm{~mL})$, 无 水硫酸钠干燥, 浓缩, 硅胶柱层析分离 $[V$ (石油醚) : $V($ 乙酸乙酯 $)=100: 1$ ] 得到棕色油状物 $1.98 \mathrm{~g} \mathbf{5}$, 收率为 77.0\%. ${ }^{1} \mathrm{H}$ NMR $\left(\mathrm{CDCl}_{3}, 400 \mathrm{MHz}\right) \delta: 5.28(\mathrm{~s}, 1 \mathrm{H}, \mathrm{H}-1)$, $5.24 \sim 5.12(\mathrm{~m}, 2 \mathrm{H}, \mathrm{H}-22, \mathrm{H}-23), 4.55(\mathrm{~d}, J=9.2 \mathrm{~Hz}, 1 \mathrm{H}$, H-7), 4.43 (d, $J=9.2 \mathrm{~Hz}, 1 \mathrm{H}, \mathrm{H}-6), 3.25$ (s, 3H, OMe), $2.67 \sim 2.54(\mathrm{~m}, 2 \mathrm{H}), 2.35 \sim 2.31(\mathrm{~m}, 1 \mathrm{H}, \mathrm{H}-2 \mathrm{~b}), 2.02 \sim$ $1.94(\mathrm{~m}, 4 \mathrm{H}), 1.87 \sim 1.84(\mathrm{~m}, 1 \mathrm{H}), 1.82 \sim 1.64(\mathrm{~m}, 4 \mathrm{H})$, $1.49 \sim 1.25(\mathrm{~m}, 7 \mathrm{H}), 1.01(\mathrm{~d}, J=6.4 \mathrm{~Hz}, 3 \mathrm{H}, \mathrm{H}-21), 0.91$ (d, $J=6.8 \mathrm{~Hz}, 3 \mathrm{H}, \mathrm{H}-28), 0.87 \sim 0.80$ (m, 7H, H-26, H-27, H-4b), 0.51 (s, 3H, H-18); ${ }^{13} \mathrm{C}$ NMR $\left(\mathrm{CDCl}_{3}, 100 \mathrm{MHz}\right) \delta$ : 152.4, 146.0, 135.4, 132.1, 116.5, 113.1, 74.3, 56.3, 56.3, 55.8, 45.2, 42.8, 40.4, 40.0, 33.1, 30.1, 29.7, 29.2, 27.7, 23.5, 22.1, 21.1, 19.9, 19.6, 18.7, 17.6, 16.8, 12.1; ESI-MS $m / z: 567.3[\mathrm{M}+\mathrm{Na}]^{+}$.

\subsection{6-甲氧基-1,10-烯-3,5-环维生素 $\mathrm{D}_{2}$ (6)的合成}

将 $5(2.85 \mathrm{~g}, 5.23 \mathrm{mmol})$, 三苯基膦 $137.07 \mathrm{mg}(0.10$ equiv.), 醋酸钯 $58.72 \mathrm{mg}$ (0.05 equiv.)投入反应瓶，用 2 $\mathrm{mL}$ DMF 完全溶解. 加入 $N, N$-二异丙基乙基胺 $1.98 \mathrm{~g}$ (3.00 equiv.), 在氮气保护下, 注入无水甲酸 $481.31 \mathrm{mg}$ (2.00 equiv.), $60{ }^{\circ} \mathrm{C}$ 水浴搅拌 $0.5 \mathrm{~h}$. TLC [ $V$ (石油醚) : $V($ 乙酸乙酯 $)=50: 1$ ] 监测至反应完全. 向瓶中注入 25 $\mathrm{mL}$ 水, 依次用乙酸乙酯萃取 $(25 \mathrm{ml} \times 3), 3 \%$ 盐酸 $(25 \mathrm{~mL} \times 2)$ 、饱和碳酸氢钠 $(25 \mathrm{~mL} \times 2)$ 、饱和食盐水洗 涤 $(25 \mathrm{~mL})$, 无水硫酸钠干燥, 浓缩, 硅胶柱层析分离 $[V$ (石油醚) $: V($ 乙酸乙酯 $)=150 ： 1$ ] 得到淡黄色油状物 $1.36 \mathrm{~g} \mathrm{6}$, 收率为 $65.7 \% .{ }^{1} \mathrm{H}$ NMR $\left(\mathrm{CDCl}_{3}, 400 \mathrm{MHz}\right) \delta$ : $5.98 \sim 5.92$ (m, 1H, H-10), 5.41 (brd, $J=4.4 \mathrm{~Hz}, 1 \mathrm{H}, \mathrm{H}-1$ ), $5.21 \sim 5.18(\mathrm{~m}, 2 \mathrm{H}, \mathrm{H}-22, \mathrm{H}-23), 4.87(\mathrm{~d}, J=8.8 \mathrm{~Hz}, 1 \mathrm{H}$, H-7), 3.88 (d, $J=9.2 \mathrm{~Hz}, 1 \mathrm{H}, \mathrm{H}-6), 3.29$ (s, 3H, OMe), 
$2.59 \sim 2.55$ (m, 2H, H-9a, H-2a), 2.30 (brd, $J=18 \mathrm{~Hz}, 1 \mathrm{H}$, $\mathrm{H}-2 \mathrm{~b}), 2.03 \sim 1.95(\mathrm{~m}, 4 \mathrm{H}), 1.88 \sim 1.83(\mathrm{~m}, 1 \mathrm{H}), 1.75 \sim$ $1.24(\mathrm{~m}, 10 \mathrm{H}), 1.05 \sim 1.01(\mathrm{~m}, 4 \mathrm{H}, \mathrm{H}-4 \mathrm{a}, \mathrm{H}-21), 0.93 \sim$ 0.89 (m, 4H, H-4b, H-28), $0.85 \sim 0.82$ (m, 6H, H-26, $\mathrm{H}-27), 0.52$ (s, 3H, H-18); ${ }^{13} \mathrm{C} \mathrm{NMR}\left(\mathrm{CDCl}_{3}, 100 \mathrm{MHz}\right) \delta$ : $142.7,135.5,134.5,131.9,127.8,119.9,78.4,56.4,60.0$, $55.9,45.3,42.8,40.4,40.3,40.0,36.0,33.0,29.4,27.8$, 24.0, 22.0, 21.5, 21.1, 19.9, 19.6, 18.4, 17.6, 11.8; ESI-MS $m / z: 419.3[\mathrm{M}+\mathrm{Na}]^{+}$.

\section{$1.71 \alpha$-羟基-6-甲氧基-19-去甲-3,5-环维生素 $\mathrm{D}_{2}$ (7)的 合成}

将 6 (500 mg, $1.26 \mathrm{mmol}$ ，固体 9-BBN $339 \mathrm{mg}$ (2.20 equiv.), 加入反应瓶, 在氮气保护下, 注入 $2 \mathrm{~mL}$ 无水四 氢呋喃, 室温摚拌 $2 \mathrm{~h}$. TLC [ $V$ (石油醚) $: V$ (乙酸乙酯 $)=$ $50: 1$ ]监测至反应完全. 加入 $1 \mathrm{~mL}$ 水, $3 \mathrm{~mL}$ 氢氧化钠水

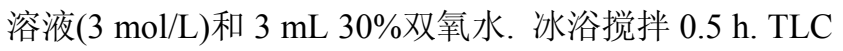
$[V($ 石油醚 $): V($ 乙酸乙酯 $)=4: 1]$ 显示反应完全. 反应 液依次用乙酸乙酯萃取 $(25 \mathrm{~mL} \times 3), 3 \%$ 盐酸 $(25 \mathrm{~mL} \times$ $2)$ 、饱和碳酸氢钠 $(25 \mathrm{~mL} \times 2)$ 、饱和食盐水洗涤 $(25 \mathrm{~mL})$, 无水硫酸钠干燥, 浓缩, 硅胶柱层析分离 $[V$ (石油醚) : $V($ 乙酸乙酯 $)=12 ： 1$ ]得到无色油状物 $183 \mathrm{mg}$ 7, 收率 为 $35.1 \% .{ }^{1} \mathrm{H} \mathrm{NMR}\left(\mathrm{CDCl}_{3}, 400 \mathrm{MHz}\right) \delta: 5.24 \sim 5.16(\mathrm{~m}$, $2 \mathrm{H}, \mathrm{H}-22, \mathrm{H}-23$ ), 4.82 (d, $J=9.2 \mathrm{~Hz}, 1 \mathrm{H}, \mathrm{H}-7$ ), $4.12 \sim 4.02$ (m, 1H, H-1), 3.97 (d, J=9.6 Hz, 1H, H-6), 3.25 (s, 3H, $\mathrm{OMe}), 2.59 \sim 2.55(\mathrm{~m}, 1 \mathrm{H}, \mathrm{H}-9 \mathrm{a}), 2.05 \sim 1.64(\mathrm{~m}, 10 \mathrm{H})$, $1.50 \sim 1.201$ (m, 9H), 1.02 (d, $J=6.4 \mathrm{~Hz}, 3 \mathrm{H}, \mathrm{H}-21), 0.92$ (d, $J=6.8 \mathrm{~Hz}, 3 \mathrm{H}, \mathrm{H}-28), 0.85 \sim 0.82(\mathrm{~m}, 6 \mathrm{H}, \mathrm{H}-26$, H-27), 0.71 (dd, $J=3.6,4.8 \mathrm{~Hz}, 1 \mathrm{H}, \mathrm{H}-4 \mathrm{a}), 0.57$ (s, 3H, $\mathrm{H}-18), 0.36$ (t, $J=4.4 \mathrm{~Hz}, 1 \mathrm{H}, \mathrm{H}-4 \mathrm{~b}) ;{ }^{13} \mathrm{C}$ NMR $\left(\mathrm{CDCl}_{3}\right.$, $100 \mathrm{MHz}) \delta: 143.9,135.5,132.0,118.6,78.4,73.0,56.0$, $56.0,55.9,45.2,42.8,40.3,40.2,38.8,37.9,33.0,31.5$, 29.4, 27.7, 23.8, 22.0, 21.1, 19.9, 19.6, 18.3, 17.6, 17.5, 12.1; ESI-MS $m / z: 437.33[\mathrm{M}+\mathrm{Na}]^{+}$.

\subsection{1 $\alpha$-羟基-19-去甲维生素 $\mathrm{D}_{2}$ (8)的合成}

将 7 (150 mg, $0.36 \mathrm{mmol}$ ) 和 $1 \mathrm{~mL}$ 冰醋酸加入反应 瓶, $60{ }^{\circ} \mathrm{C}$ 搅拌 $0.5 \mathrm{~h}$. TLC [ $V$ (石油醚 $): V($ 乙酸乙酯 $)=$ $4: 1$ ]监测至反应完全. 反应液依次用乙酸乙酯萃取 (15 $\mathrm{mL} \times 3)$, 饱和碳酸氢钠溶液 $(25 \mathrm{~mL} \times 2)$ 、饱和食盐水洗 涤 $(25 \mathrm{~mL})$, 无水硫酸钠干燥, 浓缩. 将浓缩得到的油状 物和 $2 \mathrm{~mL} 10 \% \mathrm{KOH}$ 甲醇溶液投入反应瓶, 冰浴搅拌 1 h. TLC $[V$ (石油醚) $: V($ 乙酸乙酯 $)=2.5: 1$ ] 监测至反应 完全. 反应液依次用乙酸乙酯萃取 $(25 \mathrm{~mL} \times 3), 3 \%$ 盐酸 $(25 \mathrm{~mL} \times 2)$ 、饱和碳酸氢钠 $(25 \mathrm{~mL} \times 2)$ 、饱和食盐水洗 涤 $(25 \mathrm{~mL})$, 无水硫酸钠干燥, 浓缩, 硅胶柱层析分离 $[V($ 石油醚 $): V($ 乙酸乙酯 $)=5: 1]$ 得到白色晶体粉末
$120 \mathrm{mg}$ 8, 收率为 83.3\%. ${ }^{1} \mathrm{H}$ NMR $\left(\mathrm{CDCl}_{3}, 400 \mathrm{MHz}\right) \delta$ : $6.31(\mathrm{~d}, J=11.2 \mathrm{~Hz}, 1 \mathrm{H}, \mathrm{H}-6), 5.84$ (d, $J=11.2 \mathrm{~Hz}, 1 \mathrm{H}$, $\mathrm{H}-7), 5.25 \sim 5.14(\mathrm{~m}, 2 \mathrm{H}, \mathrm{H}-22, \mathrm{H}-23), 4.16 \sim 4.09(\mathrm{~m}, 1 \mathrm{H}$, $\mathrm{H}-1), 4.09 \sim 4.01(\mathrm{~m}, 1 \mathrm{H}, \mathrm{H}-3), 2.82 \sim 2.72(\mathrm{~m}, 2 \mathrm{H}, \mathrm{H}-9 \mathrm{a}$, H-2a), $2.49 \sim 2.46(\mathrm{~m}, 1 \mathrm{H}, \mathrm{H}-4 \mathrm{a}), 2.24 \sim 2.19(\mathrm{~m}, 2 \mathrm{H})$, $2.04 \sim 1.45(\mathrm{~m}, 11 \mathrm{H}), 1.36 \sim 1.25(\mathrm{~m}, 5 \mathrm{H}), 1.01(\mathrm{~d}, J=6.8$ $\mathrm{Hz}, 3 \mathrm{H}, \mathrm{H}-21$ ), 0.92 (d, $J=6.8 \mathrm{~Hz}, 3 \mathrm{H}, \mathrm{H}-28), 0.85 \sim 0.81$ (m, 6H, H-26, H-27), 0.55 (s, 3H, H-18); ${ }^{13} \mathrm{C}$ NMR $\left(\mathrm{CD}_{3} \mathrm{OD}, 100 \mathrm{MHz}\right) \delta: 141.9,137.1,133.9,133.2,123.5$, 117.2, 67.9, 67.7, 57.8, 57.6, 46.7, 45.4, 44.4, 42.7, 41.9, $41.8,37.7,34.4,29.8,29.1,24.5,23.3,21.8,20.6,20.2$, 18.4, 12.8; ESI-MS $m / z: 423.3[\mathrm{M}+\mathrm{Na}]^{+}$.

\section{9 帕立骨化醇(9)的生物合成}

$500 \mathrm{~mL}$ 摇瓶装 $100 \mathrm{~mL}$ 培养液, $1.5 \%$ 葡萄糖, $1.5 \%$ 黄豆饼粉, $0.5 \%$ 玉米浆, $0.5 \% \mathrm{NaCl}, 0.2 \% \mathrm{CaCO}_{3}, \mathrm{pH} 7.0$. 将培养基在 $121{ }^{\circ} \mathrm{C}$ 灭菌 $20 \mathrm{~min}$. 接入菌株 CGMCC 5098 的狍子悬液 $2 \mathrm{~mL}, 27{ }^{\circ} \mathrm{C}$ 旋转, 转速 $240 \mathrm{r} / \mathrm{min}$, 培养 $4 \mathrm{~d}$. 将 $50 \mathrm{mg} 1 \alpha$-羟基-19-去甲维生素 $\mathrm{D}_{2}(8), 10 \mathrm{mg} \beta$-环糊 精, $10 \mathrm{mg}$ 吐温- 80 溶解于 $2 \mathrm{~mL}$ 乙醇, 将此混合物加入培 养液, 继续培养 $3 \mathrm{~d}$. 取出发酵液, 过滤, 滤液依次用乙 酸乙酯萃取 $(50 \mathrm{~mL} \times 3), 3 \%$ 盐酸 $(50 \mathrm{~mL} \times 2)$ 、饱和碳酸 氢钠 $(50 \mathrm{~mL} \times 2)$ 、饱和食盐水洗涤 $(50 \mathrm{~mL})$, 无水硫酸钠 干燥, 浓缩, 硅胶柱层析分离 $[V($ 石油醚 $): V($ 乙酸乙 酯 $)=2: 1$ ]得到白色晶体粉末 $31 \mathrm{mg}$ 9, 收率为 $59.6 \%$. ${ }^{1} \mathrm{H}$ NMR $\left(\mathrm{CD}_{3} \mathrm{OD}, 400 \mathrm{MHz}\right) \delta: 6.12(\mathrm{~d}, J=11.2 \mathrm{~Hz}, 1 \mathrm{H}$, H-6), 5.79 (d, $J=11.2 \mathrm{~Hz}, 1 \mathrm{H}, \mathrm{H}-7), 5.29 \sim 5.18(\mathrm{~m}, 2 \mathrm{H}$, H-22, H-23), 3.96 3.87 (m, 2H, H1, H-3), 2.76 2.72 (m, 1H, H-9a), $2.51 \sim 2.47$ (m, 1H, H-4a), $2.33 \sim 2.29(\mathrm{~m}, 1 \mathrm{H}$, $\mathrm{H}-4 \mathrm{a}), 2.14 \sim 2.04(\mathrm{~m}, 2 \mathrm{H}), 1.99 \sim 1.89(\mathrm{~m}, 4 \mathrm{H}), 1.74 \sim$ $1.16(\mathrm{~m}, 11 \mathrm{H}), 1.04$ (s, 3H, H-26), 1.00 (s, 3H, H-27), 0.94 (d, $J=6.4 \mathrm{~Hz}, 3 \mathrm{H}, \mathrm{H}-21), 0.90$ (d, $J=6.8 \mathrm{~Hz}, 3 \mathrm{H}, \mathrm{H}-28$ ), 0.49 (s, 3H, H-18); ${ }^{13} \mathrm{C}$ NMR $\left(\mathrm{CD}_{3} \mathrm{OD}, 100 \mathrm{MHz}\right) \delta$ : 132.7, 129.1, 124.6, 122.1, 114.1, 107.9, 64.0, 58.7, 58.4, 48.5, 48.3, 39.9, 37.4, 36.1, 33.3, 32.6, 32.5, 28.3, 20.5, 19.7, 19.0, 16.7, 15.2, 14.0, 12.2, 6.4, 3.4; HRMS (ESI) calcd for $\mathrm{C}_{27} \mathrm{H}_{44} \mathrm{O}_{3} \mathrm{Na}[\mathrm{M}+\mathrm{Na}]^{+}$439.3188, found 439.3191 .

\section{2 结果与讨论}

\section{1 实验路线选择}

本研究从更易获得的维生素 $\mathrm{D}_{2}$ 而不是文献 $[12,13]$ 所采用的缺乏商业来源的 25 -羟基-维生素 $\mathrm{D}_{2}$ 出发, 极 大地降低了成本, 且避免了合成过程中对 25 位羟基保 护和脱保护等一系列会影响反应产率的步骤, 通过化学 合成获得 $1 \alpha$-羟基-19-去甲维生素 $\mathrm{D}_{2}$ 后, 利用生物转化 
法实现 25 位羟基化，获得了帕立骨化醇，总收率为 $3.24 \%$.

\section{2 化合物 3 和 7 的合成}

通过第一步的着基保护得到化合物 2 之后, 本研究 摒弃了价格昂贵, 有毒有害的四氧化锇, 而选择价格便 宜, 低毒低害的高锰酸钾, 来实现双键的双差基化. 这 种方法较文献中使用的四氧化锇而言, 不仅极大地降低 了实验成本与实验操作难度, 且该步收率与文献报道相 当. 化合物 7 是通过化合物 6 进行嗍氢化氧化反应得到 的, 选用位阻较大 9-BBN 作为硼氢化试剂, 可以顺利 地实现 $1 \alpha$ 位羟基化，而不会得到 $1 \beta$ 羟基化物.

\section{3 帕立骨化醇(9)的获得}

对维生素 $\mathrm{D}$ 类似物进行生物转化差基化已有文献 报道 ${ }^{[14]}$, 目前生物制备活性维生素 $\mathrm{D}_{2}$ 或 $\mathrm{D}_{3}$, 其差基化 作用主要发生在 $1 \alpha$ 位和 25 位. 以维生素 $\mathrm{D}_{2}$ 为底物的生 物转化着基化会得到 $1 \alpha$-羟基维生素 $\mathrm{D}_{2}, 25$-羟基维生素 $\mathrm{D}_{2}$ 以及 $1 \alpha, 25$-双差基维生素 $\mathrm{D}_{2}$. 这也是为什么 25 -差基 维生素 $\mathrm{D}_{2}$ 难以大量获得的重要原因之一. 本研究采用 的策略是最后一步通过生物转化法实现 25 位着基化. 在经过七步化学合成反应得到 $1 \alpha$-羟基-19-去甲维生素 $\mathrm{D}_{2}(\mathbf{8})$ 之后, 最后一步生物转化使用的是本实验室篎选 所得的 CGMCC 5098 型放线菌株, 该菌株对 19-去甲维 生素 D 类似物具有独特的 25-羟基化活性, 转化产率为 $60 \%$, 而本工作利用生物转化法对 19-去甲维生素 D 类 似物进行 25 位着基化乃属首次报道.

\section{4 各中间体及目标化合物结构的鉴定}

合成中间体及目标物的结构均经过 ${ }^{1} \mathrm{H} \mathrm{NMR},{ }^{13} \mathrm{C}$ NMR 和 MS 表征. 目标化合物与参考文献[13]数据一致. 6-甲氧基-3,5 环维生素 $\mathrm{D}_{2}$ (2)经过高锰酸钾水溶液双着 基化之后其 ${ }^{1} \mathrm{H}$ NMR 谱图中在 $\delta 5.03$ 和 4.87 处的两个端 烯烃氢的信号消失, 在 $\delta 3.64$ 和 3.53 增加了两个二重峰
信号，为与新生成的羟基相连的亚甲基氢的信号；6-甲 氧基-1,10-烯-3,5-环维生素 $\mathrm{D}_{2}$ (6)经过嗍氢化氧化反应 之后其 ${ }^{1} \mathrm{H}$ NMR 谱图中在 $\delta 5.95$ 和 5.41 处的两个内烯烃 氢的信号消失, 在 $\delta 4.05$ 增加了一个多重峰信号, 为与 新生成的羟基相连的次甲基氢的信号；1 $\alpha$-差基-19-去甲 维生素 $\mathrm{D}_{2}(8)$ 在经过生物转化之后, 其 ${ }^{13} \mathrm{C} \mathrm{NMR}$ 谱图中 $\delta$ 在 64.0 处出现了一个峰, 为与新生成的 25 位羟基相连 的碳的信号.

\section{References}

[1] Ebert, R.; Schutze, N.; Adamski, J.; Jakob, F. Mol. Cell Endocrinol. 2006, 248, 149.

[2] Abe, E.; Miyaura, C.; Sakagami, H.; Takeda, M.; Konno, K.; Yamazaki, T.; Yoshiki, S.; Suda, T. Proc. Natl. Acad. Sci. U. S. A. 1981, 78, 4990.

[3] Yamada, S.; Shimizu, M.; Yamamoto, K. Med. Res. Rev. 2003, 23, 89.

[4] Slatopolsky, E.; Cozzolino, M.; Lu, Y.; Finch, J.; Dusso, A.; Staniforth, M.; Wein, Y.; Webster, J. Kidney Int. 2003, 63, 2020.

[5] Yang, S.; Smith, C.; Dluca, H. F. Biochim. Biophys. Acta 1993 , $1158,279$.

[6] Slatopolsky, E.; Finch, J.; Ritter, C.; Denda, M.; Morrissey, J.; Brown, A.; DeLuca, H. Am. J. Kidney Dis. 1955, $26,852$.

[7] Deluca, H. F.; Schnoes. H. K.; Perlman, K. L. US 5281731, 1994 [Chem. Abstr. 1993, 118, 124883].

[8] Deluca, H. F.; Schnoes, H. K.; Perlman, K. L. US 5086191, 1992 [Chem. Abstr. 1992, 116, 255878].

[9] Li, L.-Q.; Yue, L.-R.; Xue, J.-J.; Xie, Z.-X.; Li, Y. Chin. Sci. Bull. 2012, 57,1

[10] Ng, C.-S.; Wei, C.-P. US 007491712, 2009 [Chem. Abstr. 2009, 150, 260414].

[11] Bader, T.; Stutz, A.; Bichsel, H. U.; Stutz, A.; Fu, C.-C. WO 2010009879, 2010 [Chem. Abstr. 2010, 152, 19236].

[12] Perlman, K. L.; Sicinski R. R.; Schnoes, H. K.; DeLuca, H. F. Tetrahedron Lett. 1990, 31, 1823.

[13] Toyoda, A.; Nagai, H.; Yamada, T.; Moriguchi, Y.; Abe, J.; Tsuchida, T.; Nagasawa; K. Tetrahedron 2009, 65, 10002.

[14] Takeda, K.; Asou, T.; Matsuda, A.; Kimura, K.; Okamura, K.; Okamoto, R.; Sasaki, J.; Adachi, T.; Omura, S. J. Ferment. Bioeng. 1994, $78,380$. 